\title{
Electric Stimulation of Ear Reduces the Effect of Toll-Like Receptor 4 Signaling Pathway on Kainic Acid-Induced Epileptic Seizures in Rats
}

\author{
En-Tzu Liao, ${ }^{1}$ Yi-Wen Lin $(1),{ }^{2,3}$ Chun-Ping Huang, ${ }^{3}$ \\ Nou-Ying Tang, ${ }^{4}$ and Ching-Liang Hsieh $\mathbb{B}^{2,3,5,6}$ \\ ${ }^{1}$ Graduate Institute of Chinese Medicine, College of Chinese Medicine, China Medical University, Taichung 40402, Taiwan \\ ${ }^{2}$ Graduate Institute of Acupuncture Science, College of Chinese Medicine, China Medical University, Taichung 40402, Taiwan \\ ${ }^{3}$ Research Center for Chinese Medicine and Acupuncture, China Medical University, Taichung 40402, Taiwan \\ ${ }^{4}$ School of Chinese Medicine, College of Chinese Medicine, China Medical University, Taichung 40402, Taiwan \\ ${ }^{5}$ Graduate Institute of Integrated Medicine, College of Chinese Medicine, China Medical University, Taichung 40402, Taiwan \\ ${ }^{6}$ Department of Chinese Medicine, China Medical University Hospital, Taichung 40447, Taiwan
}

Correspondence should be addressed to Ching-Liang Hsieh; clhsieh@mail.cmuh.org.tw

Received 19 October 2017; Revised 10 January 2018; Accepted 18 January 2018; Published 26 February 2018

Academic Editor: Eiichi Kumamoto

Copyright (C) 2018 En-Tzu Liao et al. This is an open access article distributed under the Creative Commons Attribution License, which permits unrestricted use, distribution, and reproduction in any medium, provided the original work is properly cited.

\begin{abstract}
Epilepsy is a common clinical syndrome with recurrent neuronal discharges in the temporal lobe, cerebral cortex, and hippocampus. Clinical antiepileptic medicines are often ineffective or of little benefit in $30 \%$ of epileptic patients and usually cause severe side effects. Emerging evidence indicates the crucial role of inflammatory mediators in epilepsy. The current study investigates the role of toll-like receptor 4 (TLR4) and its underlying mechanisms in kainic acid- (KA-) induced epileptic seizures in rats. Experimental KA injection successfully initiated an epileptic seizure accompanied by increased expression of TLR4 in the prefrontal cortex, hippocampus, and somatosensory cortex. In addition, calcium-sensitive phosphorylated $\mathrm{Ca}^{2+} /$ calmodulin-dependent protein kinase II ( $\mathrm{pCaMKII} \alpha)$ increased after the initiation of the epileptic seizure. Furthermore, downstream-phosphorylated signalregulated kinase (ERK), c-Jun $\mathrm{NH}_{2}$-terminal protein kinase (JNK), and p38 kinase simultaneously increased in these brain areas. Moreover, the transcriptional factor phosphorylated nuclear factor- $\kappa \mathrm{B}(\mathrm{pNF}-\kappa \mathrm{B})$ increased, suggesting that nucleus transcription was affected. Furthermore, the aforementioned molecules decreased by an electric stimulation (ES) of either $2 \mathrm{~Hz}$ or $15 \mathrm{~Hz}$ of the ear in the three brain areas. Accordingly, we suggest that ES of the ear can successfully control epileptic seizures by regulating the TLR4 signaling pathway and has a therapeutic benefit in reducing epileptic seizures.
\end{abstract}

\section{Introduction}

Temporal lobe epilepsy is a neurological disease causing abnormal discharges in the brain, particularly in the cortex and hippocampus. Epilepsy is defined as an abnormal condition of brain imbalance, with unpredictable electrical discharges and seizures. According to clinical studies, $30 \%$ of epileptic patients undergo uncontrolled epileptic seizures owing to ineffective antiepileptic drugs. In general, several antiepileptic drugs serve as antiexcitatory or enhanced inhibitory agents to depress seizure occurrence. Accordingly, these medicines have severe side effects on cognition and memory $[1,2]$.
Epilepsy is the result of an imbalance between excitatory and inhibitory function and can be induced in animal models by overactivation of excitatory neurotransmitter receptors through a kainic acid (KA) injection, which is utilized to initiate epileptic seizures [3]. Another method to induce epileptic seizures involves blocking the inhibitory $\mathrm{GABA}_{\mathrm{A}}$ receptors, and this can be performed using pilocarpine [4]. Increase of GABA, including gabapentin and sodium valproate, was clinically used for epileptic seizure control with little effectiveness and numerous side effects.

Toll-like receptors (TLR) are mainly located on the cell membrane, but TLR3 and TLR7 are located in the endosomal 
compartment [5]. TLR2, TLR3, and TLR4 are majorly expressed in microglia, astrocytes, and neurons, and their expression is decreased because of physiological conditions. In pathology-related epileptic seizures, TLRs are increased [6-9]. Activation of TLR2 triggers the signaling pathway for myeloid differentiation factor 88 (MyD88) and further activates nuclear factor kappa-light-chain-enhancer of activated $\mathrm{B}$ cells $(\mathrm{NF}-\kappa \mathrm{B})$ in the nucleus to increase proinflammatory mediators [10]. Several studies have demonstrated that TLR4 is crucial for regulating inflammatory responses [11-13]. TLR4 is reported to recognize both exogenous pathogenassociated and endogenous damage-associated molecular patterns. TLR4 is also reported to act as a receptor, that is, a marker of microglial activation in central nervous system (CNS) inflammation $[14,15]$.

Acupuncture has been used in Asia for thousands of years to treat diseases. A number of studies have indicated that electroacupuncture (EA) can be used to treat stroke-induced dementia [16], epilepsy [3], body weight control [17], Parkinson's disease [18], depression [19], and pain [20, 21]. Initially, scientists determined that acupuncture treatment worked through the release of endogenous opiates [22], serotonin [23], and adenosine [24]. The well-known mechanism of acupuncture analgesia involves the release of opiates in CNS [22] and adenosine in the peripheral nervous system (PNS) [24]. A recent study showed that long-term electric stimulation (ES) of the ear significantly reduces inflammatory mediators in CA1 of the hippocampus in KA-induced epileptic seizure in rats [3]. In the current study, we hypothesized that TLR4 and related molecules are crucial for epileptic seizure in rats. ES of the ear is effective in treating KA-induced epilepsy seizure by regulating TLR4 and related pathways in the rat brain.

\section{Materials and Methods}

2.1. Animals. Male Sprague-Dawley (SD) rats weighing 200$300 \mathrm{~g}$ were purchased from BioLASCO (BioLASCO Taiwan Co., Ltd) and hosted in the animal center of China Medical University (CMU). A 12-12 h light-dark cycle was maintained, and the room temperature was controlled at $25^{\circ} \mathrm{C}$. Adequate food and water were provided. The Animal Care and Use Committee of CMU approved the use of these animals. In addition, all procedures were performed according to the Guide for the Use of Laboratory Animals (National Academy Press).

2.2. Epileptic Seizure Rat Model. Thirty SD rats were placed in a stereotaxic apparatus in a prone position under isoflurane (Aerrane, Canada) anesthesia administered through a vaporizing system (MATRX VIP 3000, Midmark, USA). Hair from the rats' scalp was cut using surgical scissors, and a surgical knife was used to incise the scalp at the midline to expose the skull. Stainless steel screw electrodes, which were placed on the dura above the bilateral sensorimotor cortices, served as recording electrodes. A reference electrode was placed at the frontal sinus for electroencephalogram (EEG) recordings. Bipolar electrical wires were passed through the subcutaneous tissue and around the neck muscles for electromyogram (EMG) recordings. The electrodes were plugged into a conductor, which was fixed to the skull with dental acrylic cement. These electrodes were then connected to EEG- and EMG-monitoring machines (MPIOOWSW, BIOPAC Systems, Inc., CA, USA). Epileptic seizure behaviors were confirmed using a video-recording epileptic behavioral analysis system (SeizureScan, Clever Sys., Inc., Virginia, USA), and both EEG and EMG findings were recorded during a conscious and free-moving state for at least 4 days after electrode implantation. On EEG recordings, intraperitoneal injection (i.p.) of KA (12 mg/kg) was observed to mainly induce epileptic seizure behaviors, namely, wet-dog behavior, facial myoclonia, paw tremors, and epileptiform discharges. The epileptic seizures were confirmed on the observation of behavioral changes, including wet-dog shakes, paw tremors, and facial myoclonia in a freely moving and conscious state and on that of epileptiform discharges based on EEG recordings. Rats exhibiting more than 250 wet-dog shakes and more than 100 facial myoclonia plus paw tremors were selected. Epileptic seizure behaviors were observed on EEG and EMG recordings at $15 \mathrm{~min}$ before and $3 \mathrm{~h}$ after the KA injection. We followed the methods of Liao et al., 2017 [3].

2.3. Grouping. The rats were randomly divided into five experimental groups, and each group contained six rats as follows: (1) control group, in which the rats were peritoneally injected with phosphate buffer solution (PBS); (2) KA group, in which the rats were injected with KA (12 mg/kg i.p.); (3) $2 \mathrm{~Hz}$ ES group, in which the rats received $2 \mathrm{~Hz}$ ES (using clip electrodes, with the cathode at the ear apex and anode at the ear lobe; stimulus frequency: $2 \mathrm{~Hz}$; stimulus intensity: visual ear twitch; stimulus duration: $20 \mathrm{~min} /$ day, with each ear receiving the stimulus for $10 \mathrm{~min}$ alternately); (4) $15 \mathrm{~Hz}$ ES group, in which the rats received $15 \mathrm{~Hz}$ ES (using clip electrodes, with the cathode placed at the ear apex and anode at the ear lobe; stimulus frequency: $15 \mathrm{~Hz}$; stimulus intensity: visual ear twitch; stimulus duration: $20 \mathrm{~min} /$ day, with each ear receiving the stimulus for 10 min alternately); (5) sham group, in which the clip electrodes were connected to an electric stimulator without electric charge. ES was applied for 3 days per week for $20 \mathrm{~min} /$ day for 3 weeks, starting from the day following the KA injection. All the rats were sacrificed at 3 weeks, and their brains were removed.

2.4. Western Blot Analysis. Following brain extraction, the frontal cortex, hippocampus, and somatosensory cortex were immediately excised for protein extraction. The total protein was prepared by homogenizing the hippocampi for $1 \mathrm{~h}$ at $4^{\circ} \mathrm{C}$ in a lysis buffer containing $20 \mathrm{mmol} / \mathrm{L}$ of imidazole: $\mathrm{HCl}$ ( $\mathrm{pH}$ 6.8), $100 \mathrm{mmol} / \mathrm{L}$ of $\mathrm{KCl}, 2 \mathrm{mmol} / \mathrm{L}$ of $\mathrm{MgCl}_{2}, 20 \mathrm{mmol} / \mathrm{L}$ of ethyleneglycoltetraacetic acid ( $\mathrm{pH} 7.0), 300 \mathrm{mmol} / \mathrm{L}$ of sucrose, $1 \mathrm{mmol} / \mathrm{L}$ of $\mathrm{NaF}, 1 \mathrm{mmol} / \mathrm{L}$ of sodium vanadate, $1 \mathrm{mmol} / \mathrm{L}$ of sodium molybdate, $0.2 \%$ Triton X-100, and a proteinase inhibitor cocktail. From each sample, $30 \mu \mathrm{g}$ protein was extracted and analyzed through a bicinchoninic acid protein assay. The protein was subjected to $10 \%-15 \%$ sodium dodecyl sulfate-tris-glycine gel electrophoresis and was transferred to a nitrocellulose membrane. The membrane was blocked with $5 \%$ nonfat milk in TBST buffer $(10 \mathrm{mmol} / \mathrm{L}$ of Tris, $\mathrm{pH} 7.5 ; 100 \mathrm{mmol} / \mathrm{L}$ of $\mathrm{NaCl}$; and $0.1 \%$ Tween 20 ) and 
incubated overnight at $4^{\circ} \mathrm{C}$ with the primary antibodies (antiTLR4, anti-pCaMKII $\alpha$, anti-pERK, anti-pp38, anti-pJNK, and anti-pNF $\kappa \mathrm{B}$ ) in TBST containing bovine serum albumin. Peroxidase-conjugated antibody $(1: 500)$ was used as the secondary antibody. The membrane was assessed using the ECL-Plus protein detection kit. We followed the methods of Liao et al., 2017 [3].

2.5. IHC Staining. The rats were anesthetized with isoflurane and then intracardially perfused with saline. The brains were removed and postfixed in the same fixative overnight at $4^{\circ} \mathrm{C}$. After briefly washing with PBS, the brains were transferred to a $30 \%$ sucrose solution in $0.01 \mathrm{M}$ PBS for cryoprotection, and coronal sections containing the hippocampal area were cut into $16 \mu \mathrm{m}$ thick slices through cryosectioning. The sections were preincubated for $10 \mathrm{~min}$ at room temperature with $10 \%$ normal goat serum in PBS to avoid nonspecific binding. The sections were incubated overnight at $4^{\circ} \mathrm{C}$ in PBS containing the primary antibodies (anti-TLR4, anti-pERK, and anti-pNF $\kappa$ B). The sections were subsequently incubated with the biotinylated-conjugated secondary antibody (diluted at 1:200; Vector, Burlingame, CA 94010, USA) for $10 \mathrm{~min}$ at room temperature, followed by incubation with avidinhorseradish peroxidase complex (ABC kit, Genemed, USA). The sections were then visualized using $3,3^{\prime}$-diaminobenzidine as a chromogen. During the incubation steps, the sections were washed with PBS thrice for 10 min per cycle. The stained hippocampus slices were sealed under coverslips and then examined for the presence of immune-positive hippocampal neurons using a microscope (Olympus, BX-51, Japan) with a $40 \mathrm{x}$ numerical aperture $(\mathrm{NA}=1.4)$ objective. We followed the methods of Liao et al., 2017 [3].

2.6. Statistical Analysis. All data are presented as mean \pm standard deviation. Statistical significance among the control, $\mathrm{KA}, 2 \mathrm{~Hz} \mathrm{ES}, 15 \mathrm{~Hz} \mathrm{ES}$, and sham groups was analyzed through one-way ANOVA, followed by Tukey's post hoc test. A $p$ value of $<0.05$ was considered statistically significant.

\section{Results}

3.1. Effect of Ear ES on the Levels of TLR4 in Prefrontal Cortex of KA-Induced Epileptic Seizure Rats. We first used the western blot technique to investigate TLR4 and related signaling pathways in the frontal cortex. Our results indicated that TLR4 increased in KA-induced epileptic rat prefrontal cortex (Figure 1(a), 149.67\% $\pm 14.16 \%, p<0.05, n=6$ ). The potentiation was reversed by either $2 \mathrm{~Hz}$ ES (Figure 1(a), $103.35 \% \pm 10.29 \%, p<0.05, n=6$ ) or $15 \mathrm{~Hz}$ ES (Figure $1(\mathrm{a})$, $102.72 \% \pm 10.27 \%, p<0.05, n=6)$. However, we did not observe this phenomenon in sham controls (Figure 1(a), 146.18\% $\pm 17.45 \%, p>0.05, n=6)$. TLR4 activation can further initiate $\mathrm{Ca}^{2+}$ influx into cell plasma. We then checked the $\mathrm{Ca}^{2+}$ mediated second messenger pathway in rat prefrontal cortex. We showed that pCaMKII $\alpha$ significantly increased by KA injection (Figure 1(b), 156.28\% $\pm 31.97 \%, p<0.05, n=$ $6)$. This phenomenon was reversed by $2 \mathrm{~Hz}$ ES (Figure 1(b), $90.62 \% \pm 21.13 \%, p<0.05, n=6$ ) and $15 \mathrm{~Hz}$ ES (Figure 1(b),
95.41\% $\pm 17.53 \%, p<0.05, n=6$ ), except in sham controls (Figure 1(b), $139.26 \% \pm 21.73 \%, p>0.05, n=6$ ). We also checked the serial downstream molecules such as the MAPK subfamily pERK, pp38, and pJNK. We found that immunopositive signals of $\mathrm{pERK}, \mathrm{pp} 38$, and $\mathrm{pJNK}$ were increased in the KA group (Figures 1(c)-1(e), 157.52\% $\pm 13.28 \%$, $155.22 \% \pm 16.59 \%, 129.59 \% \pm 17.04 \%, p<0.05, n=6)$. All the results were reversed by $2 \mathrm{~Hz}$ ES (Figures $1(\mathrm{c})-1(\mathrm{e})$, $102.15 \% \pm 10.21 \%, 115.73 \% \pm 10.02 \%, 88.63 \% \pm 8.99 \%, p<$ $0.05, n=6$ ) and $15 \mathrm{~Hz}$ ES (Figures 1(c)-1(e), $112.02 \%$ $\pm 12.77 \%, 107.51 \% \pm 7.86 \%, 96.16 \% \pm 10.35 \%, p<0.05, n=$ 6), except in sham controls (Figures 1(c)-1(e), $160.89 \%$ $\pm 12.76 \%, 139.36 \% \pm 12.96 \%, 119.05 \% \pm 12.01 \%, p>0.05, n=$ $6)$. To investigate the transcriptional factor, we examined the level of nucleus factor $\mathrm{pNF} \kappa \mathrm{B}$. Our data demonstrated that $\mathrm{pNF} \kappa \mathrm{B}$ increased in the prefrontal cortex of epileptic rats (Figure 1(f), 143.81\% $\pm 25.04 \%, p<0.05, n=6$ ). These results were reversed by $2 \mathrm{~Hz}$ ES (Figure 1(f), $84.61 \%$ $\pm 16.78 \%, p<0.05, n=6$ ) or $15 \mathrm{~Hz}$ ES (Figure 1(f), $87.59 \%$ $\pm 19.7 \%, p<0.05, n=6$ ), except in sham controls (Figure 1(f), $126.4 \% \pm 26.34 \%, p>0.05, n=6)$.

3.2. Effect of Ear ES on the Levels of TLR4 in Hippocampus of KA-Induced Epileptic Seizure Rats. We then showed that TLR4 increased in the hippocampus of KA-induced epileptic rats (Figure 2(a), 124.45\% $\pm 7.25 \%, p<0.05, n=6$ ). This result was reversed by $2 \mathrm{~Hz}$ ES (Figure 2(a), 92.76\% $\pm 7.39 \%$, $p<0.05, n=6$ ) and $15 \mathrm{~Hz}$ ES (Figure 2(a), 95.91\% $\pm 7.75 \%$, $p<0.05, n=6$ ), except in sham controls (Figure 2(a), $137.24 \% \pm 10.54 \%, p>0.05, n=6)$. We also showed that $\mathrm{pCaMKII} \alpha$ further increased by KA injection (Figure 2(b), $135.53 \% \pm 12.53 \%, p<0.05, n=6)$ and decreased by $2 \mathrm{~Hz}$ ES (Figure 2(b), 95.27\% $\pm 7.63 \%, p<0.05, n=6$ ) and $15 \mathrm{~Hz}$ ES (Figure 2(b), 94.76\% $\pm 7.17 \%, p<0.05, n=6$ ), except in sham controls (Figure 2(b), 142.36\% $\pm 18.66 \%, p>0.05, n=$ $6)$. In addition, serial downstream molecules, such as pERK, pp38, and pJNK, significantly increased in the KA group (Figures 2(c)-2(e), $167.18 \% \pm 37.36 \%, 140.88 \% \pm 17.74 \%, 155.81 \%$ $\pm 28.37 \%, p<0.05, n=6)$. These results were then attenuated by $2 \mathrm{~Hz}$ ES (Figures 2(c)-2(e), 95.07\% $\pm 21.24 \%, 105.32 \%$ $\pm 17.18 \%, 83.93 \% \pm 17.58 \%, p<0.05, n=6)$ or $15 \mathrm{~Hz}$ ES (Figures 2(c)-2(e), 100.52\% $\pm 26.75 \%, 105.36 \% \pm 19.71 \%$, $89.31 \% \pm 16.45 \%, p<0.05, n=6$ ), except in sham controls (Figures 2(c)-2(e), 150.55\% $\pm 21.37 \%, 140.69 \% \pm 25.59 \%$, $131.58 \% \pm 12.07 \%, p>0.05, n=6)$. Furthermore, we demonstrated that $\mathrm{pNF} \kappa \mathrm{B}$ increased in the hippocampus of epileptic rats (Figure 2(f), $134.59 \% \pm 15.12 \%, p<0.05, n=6$ ). The potentiation was attenuated by both $2 \mathrm{~Hz}$ ES (Figure 2(f), $102.94 \% \pm 10.27 \%, p<0.05, n=6$ ) and $15 \mathrm{~Hz}$ ES (Figure 2(f), $109.48 \% \pm 9.42 \%, p<0.05, n=6)$, except in sham controls (Figure 2(f), $138.48 \% \pm 16.27 \%, p>0.05, n=6$ ).

3.3. Effect of Ear ES on the Levels of TLR4 in Somatosensory Cortex of KA-Induced Epileptic Seizure Rats. We further checked if the TLR4 signaling pathway was involved in the somatosensory cortex and found that TLR4 increased in the somatosensory cortex of the epileptic rats (Figure 3(a), $132.07 \% \pm 8.91 \%, p<0.05, n=6$ ). This phenomenon was 


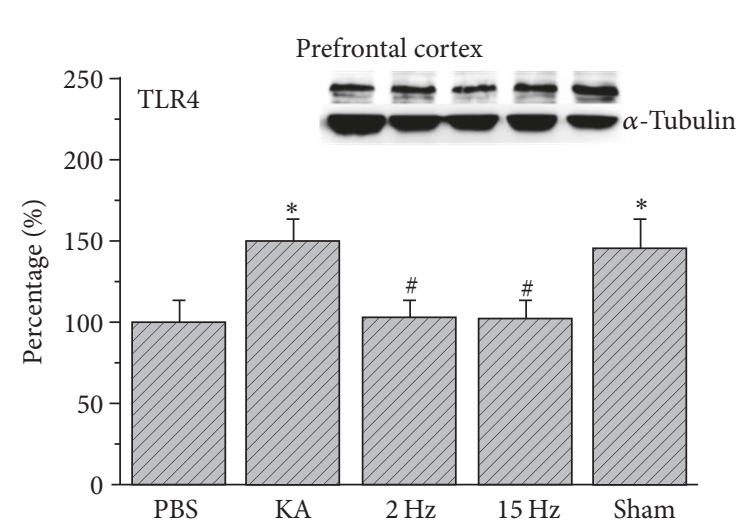

(a)

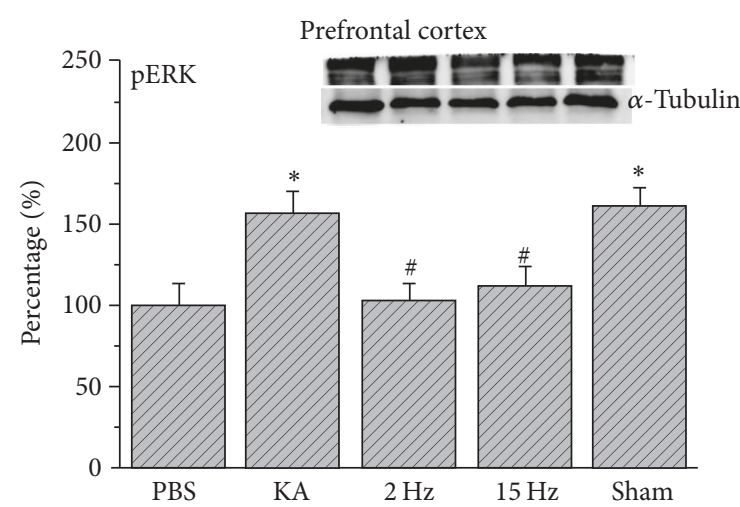

(c)

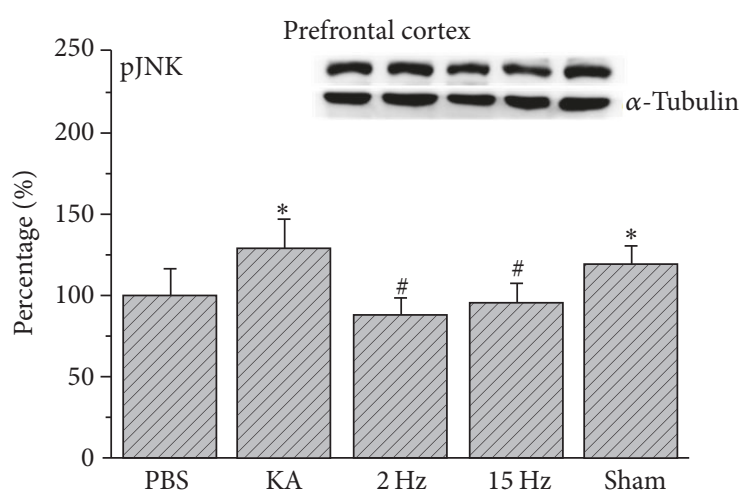

(e)

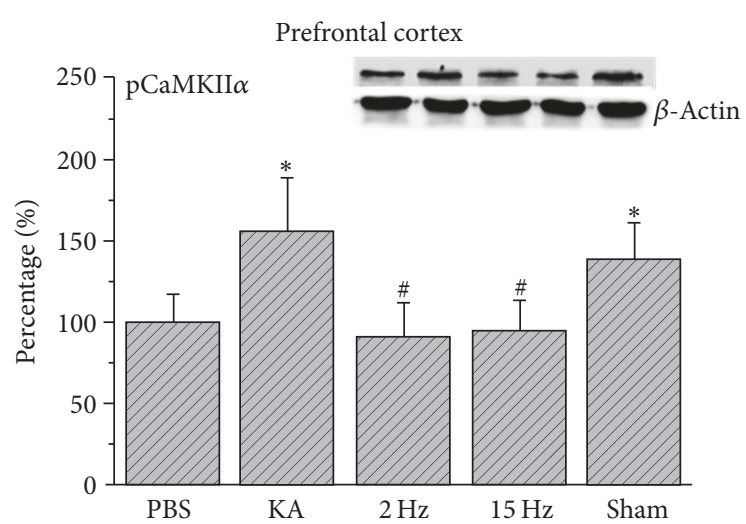

(b)

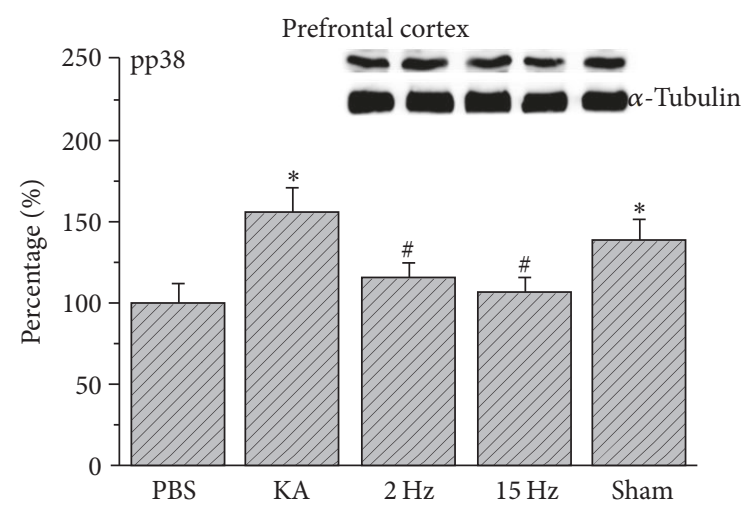

(d)

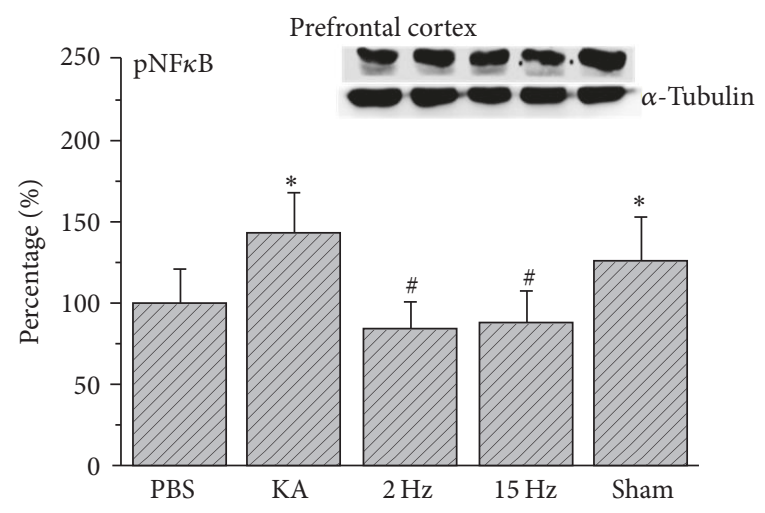

(f)

FIGURE 1: Expression levels of TLR4 and related molecular proteins in the prefrontal cortex. (a) TLR4, (b) pCaMKII $\alpha$, (c) pERK, (d) pp38, (e) $\mathrm{pJNK}$, and (f) $\mathrm{pNF} \kappa \mathrm{B}$ expression levels in the prefrontal cortex of the PBS, KA, $2 \mathrm{~Hz}, 15 \mathrm{~Hz}$, and sham ES groups (from left to right). $\mathrm{PBS}=$ phosphate-buffered saline; $\mathrm{KA}=$ kainic acid-induced epileptic rats; $2 \mathrm{~Hz} \mathrm{ES}=2 \mathrm{~Hz}$ electrical stimulation of the ear; $15 \mathrm{~Hz} \mathrm{ES}=15 \mathrm{~Hz}$ electrical stimulation of the ear; sham $=$ sham-operated electrical stimulation of the ear. ${ }^{*} p<0.05$ compared with the control group. ${ }^{\#} p<0.05$ compared with the KA group. The western blot bands at the top indicate the target protein. The lower bands correspond to the internal controls ( $\beta$-actin or $\alpha$-tubulin).

reversed by $2 \mathrm{~Hz}$ ES (Figure 3(a), 102.42\% $\pm 10.69 \%$, $p<$ $0.05, n=6$ ) and $15 \mathrm{~Hz}$ ES (Figure $3(\mathrm{a}), 103.57 \% \pm 7.79 \%$, $p<0.05, n=6$ ), except in sham controls (Figure 3(a), $129.75 \% \pm 10.02 \%, p>0.05, n=6)$. We further determined that pCaMKII $\alpha$ increased in epileptic rats (Figure 3(b), $129.45 \% \pm 17.92 \%, p<0.05, n=6$ ), and this increase was reduced by both $2 \mathrm{~Hz}$ ES (Figure 3(b), $94.55 \% \pm 12.38 \%, p<$ $0.05, n=6$ ) and $15 \mathrm{~Hz}$ ES (Figure $3(\mathrm{~b}), 98.77 \% \pm 14.92 \%$, $p<0.05, n=6$ ), except in sham controls (Figure 3(b), $134.73 \% \pm 25.33 \%, p>0.05, n=6$ ). Similarly, pERK, pp38, and pJNK also increased in the KA group (Figures 3(c)-3(e), $134.69 \% \pm 13.49 \%, 153.43 \% \pm 12.26 \%, 139.17 \% \pm 11.44 \%, p<$ $0.05, n=6$ ), and this increase was reversed by both $2 \mathrm{~Hz}$ ES (Figures 3(c)-3(e), 100.69\% $\pm 10.09 \%, 112.83 \% \pm 6.03 \%$, $102.01 \% \pm 7.47 \%, p<0.05, n=6$ ) and $15 \mathrm{~Hz}$ ES (Figures $3(\mathrm{c})-3(\mathrm{e}), 101.32 \% \pm 7.82 \%, 105.35 \% \pm 8.18 \%, 103.92 \%$ 


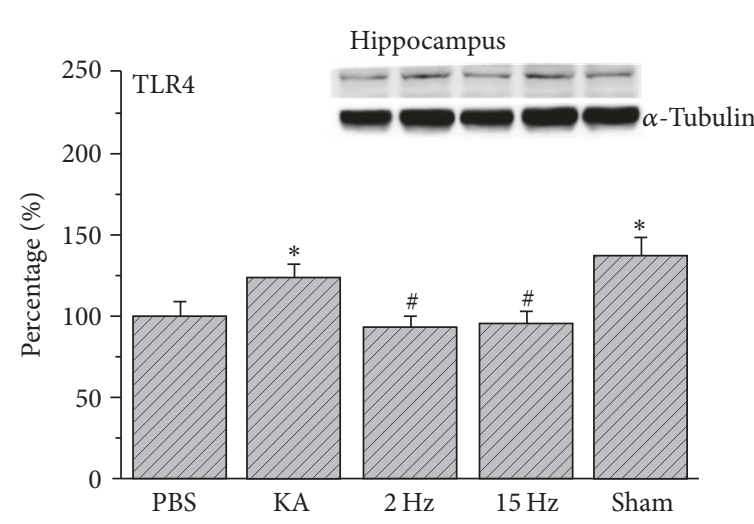

(a)

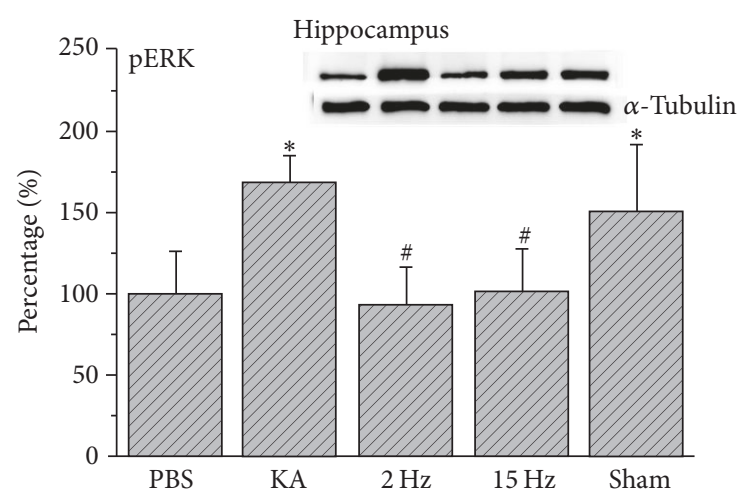

(c)

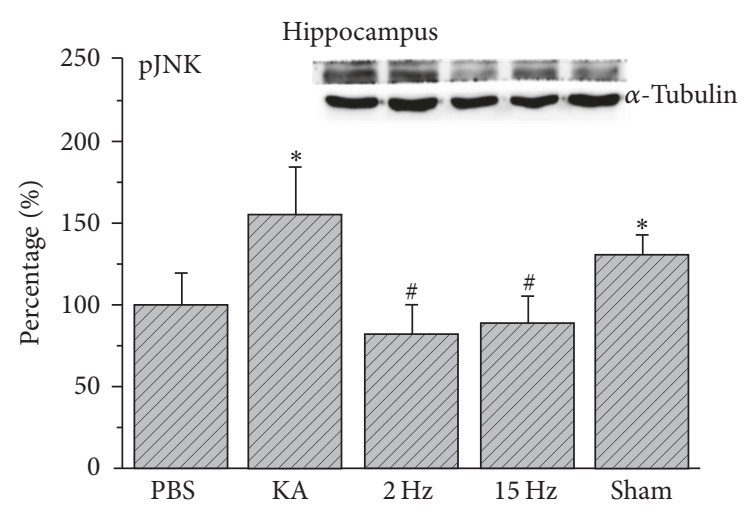

(e)

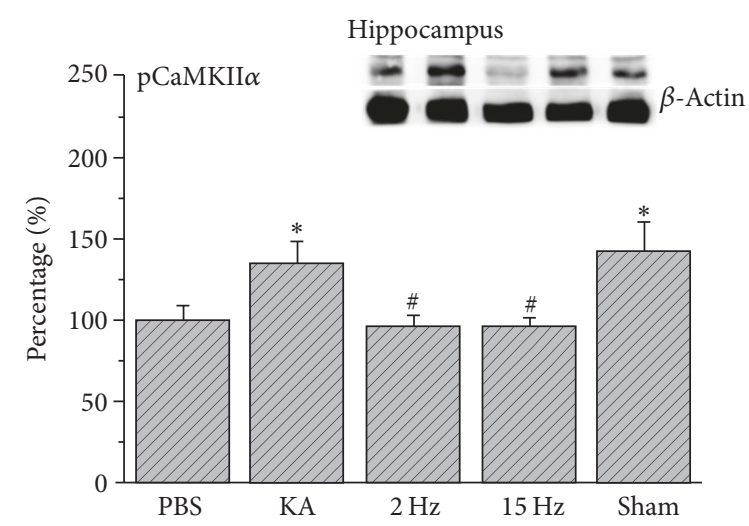

(b)

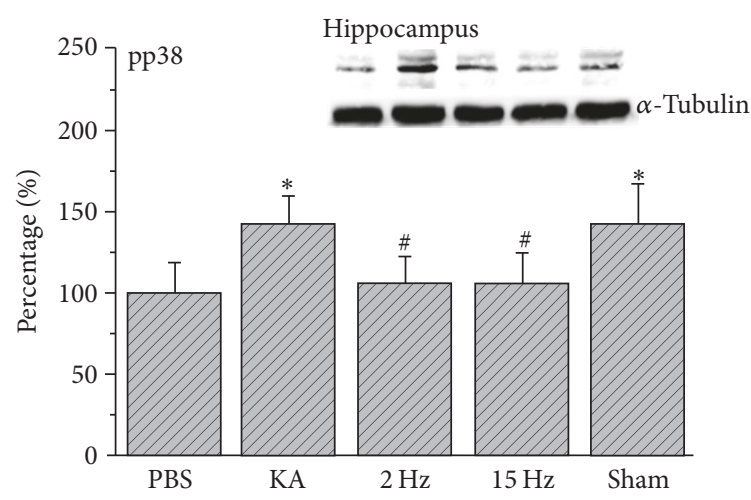

(d)

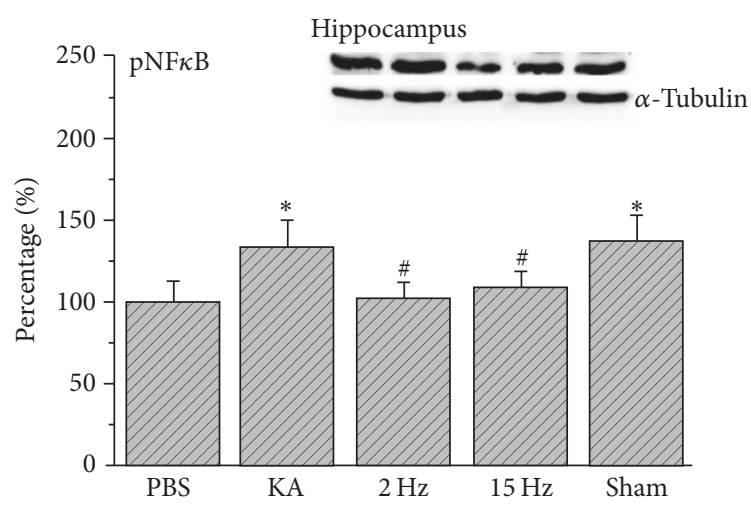

(f)

FIGURE 2: Expression levels of TLR4 and related molecular proteins in the hippocampus. (a) TLR4, (b) pCaMKII $\alpha$, (c) pERK, (d) pp38, (e) pJNK, and (f) pNF $\kappa B$ expression levels in the hippocampus of the PBS, KA, $2 \mathrm{~Hz}, 15 \mathrm{~Hz}$, and sham ES groups (from left to right). PBS = phosphate-buffered saline; KA = kainic acid-induced epileptic rats; $2 \mathrm{~Hz}$ ES $=2 \mathrm{~Hz}$ electrical stimulation of the ear; $15 \mathrm{~Hz} \mathrm{ES}=15 \mathrm{~Hz}$ electrical stimulation of the ear; sham $=$ sham-operated electrical stimulation of the ear. ${ }^{*} p<0.05$ compared with the control group. ${ }^{*} p<0.05$ compared with the KA group. The western blot bands at the top indicate the target protein. The lower bands correspond to the internal controls $(\beta$-actin or $\alpha$-tubulin).

$\pm 9.62 \%, p<0.05, n=6$ ), except in sham controls (Figures 3(c)-3(e), $126.57 \% \pm 8.52 \%, 138.17 \% \pm 8.02 \%, 129.19 \pm$ $18.78 \%, p>0.05, n=6)$. Furthermore, we determined that $\mathrm{pNF} \kappa \mathrm{B}$ increased in the somatosensory cortex of epileptic rats (Figure 3(f), 143.11\% $\pm 14.24 \%, p<0.05, n=6$ ), and this potentiation was attenuated by both $2 \mathrm{~Hz}$ ES (Figure 3(f), $91.13 \% \pm 8.55 \%, p<0.05, n=6$ ) and $15 \mathrm{~Hz}$ ES (Figure 3(f), $95.16 \% \pm 9.84 \%, p<0.05, n=6)$, except in sham controls (Figure 3(f), $121.05 \pm 10.12 \%, p>0.05, n=6$ ).
3.4. Effect of Ear ES on Immunohistochemistry Analysis of TLR4-pERK-pNFkB Expression in Hippocampus and Somatosensory Cortex. Immunohistochemical staining, visualized in brown color, demonstrated that TLR4 expression level was expressed in control rat hippocampus and somatosensory cortex, which further increased in KA-induced epileptic rats. The overexpression of TLR4 was reversed by both $2 \mathrm{~Hz}$ ES and $15 \mathrm{~Hz}$ ES, except in sham controls (Figure 4). A similar pattern was observed in pERK expression; we found that 

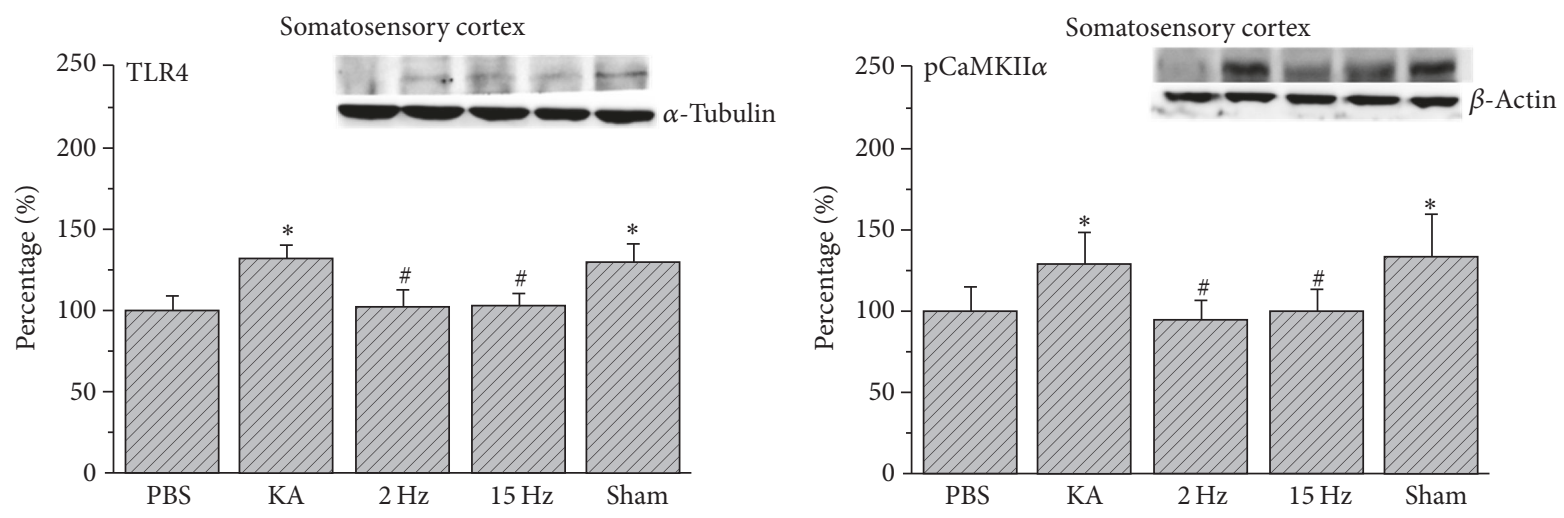

(a)
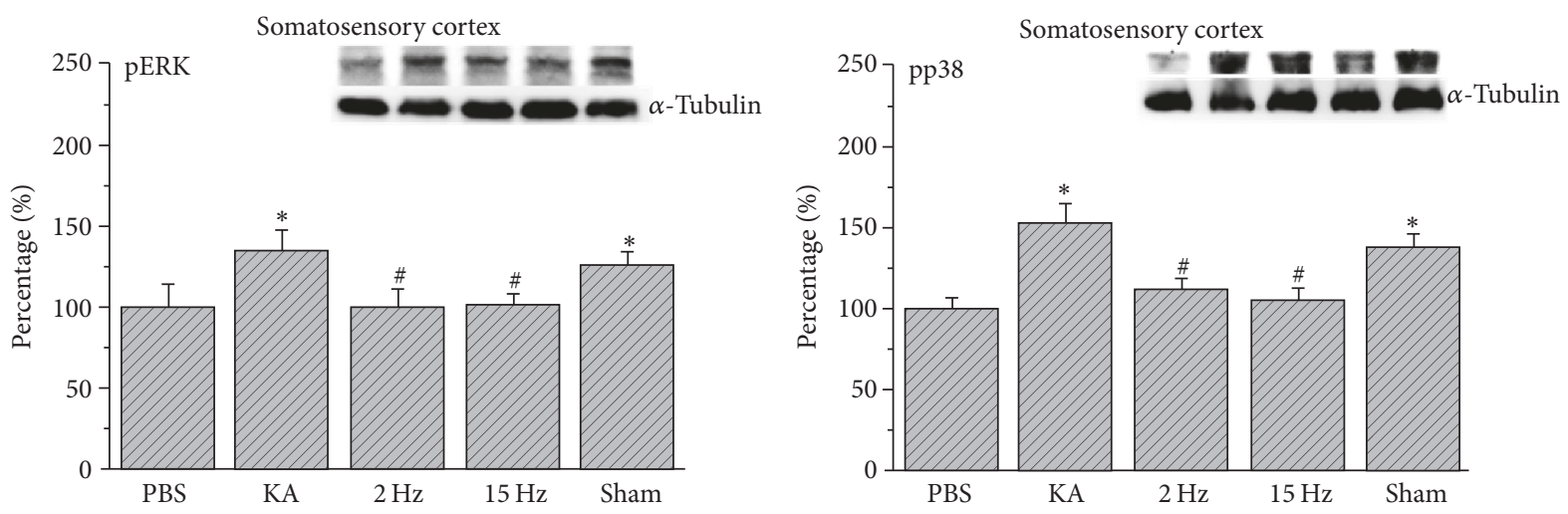

(c)

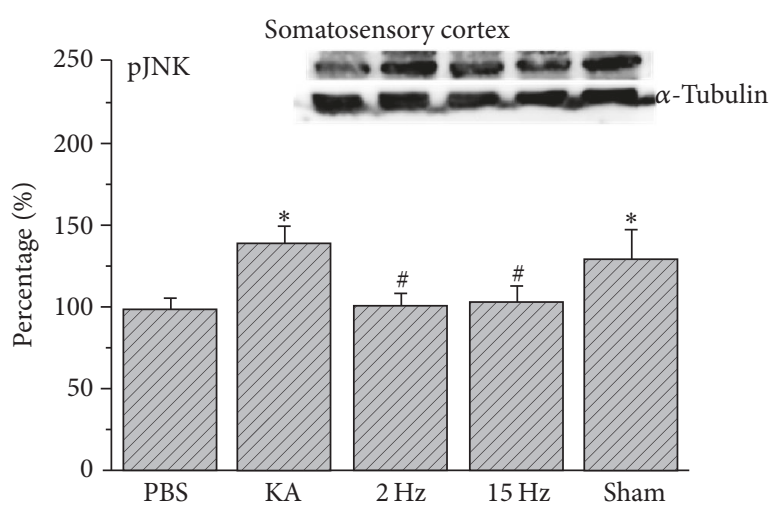

(e)

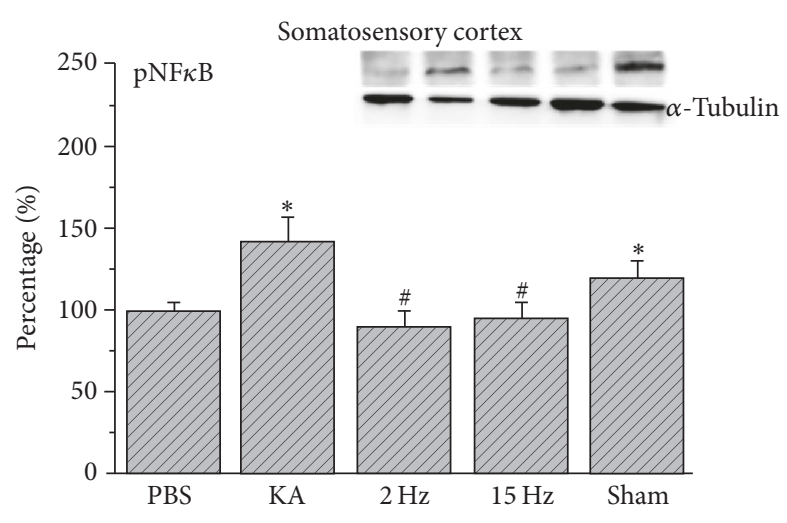

(f)

FiguRE 3: Expression levels of TLR4 and related molecular proteins in the somatosensory cortex. (a) TLR4, (b) pCaMKII $\alpha$, (c) pERK, (d) pp38, (e) pJNK, and (f) pNF $\mathrm{B}$ expression levels in the somatosensory cortex of the PBS, KA, $2 \mathrm{~Hz}, 15 \mathrm{~Hz}$, and sham ES groups (from left to right). $\mathrm{PBS}=$ phosphate-buffered saline; $\mathrm{KA}=$ kainic acid-induced epileptic rats; $2 \mathrm{~Hz} \mathrm{ES}=2 \mathrm{~Hz}$ electrical stimulation of the ear; $15 \mathrm{~Hz} \mathrm{ES}=$ $15 \mathrm{~Hz}$ electrical stimulation of the ear; sham = sham-operated electrical stimulation of the ear. ${ }^{*} p<0.05$ compared with the control group. ${ }^{\#} p<0.05$ compared with the KA group. The western blot bands at the top indicate the target protein. The lower bands correspond to the internal controls ( $\beta$-actin or $\alpha$-tubulin).

pERK increased after KA injection, and this was reversed by both $2 \mathrm{~Hz}$ ES and $15 \mathrm{~Hz}$ ES, except in sham controls (Figure 5). We further determined that $\mathrm{pNF} \kappa \mathrm{B}$ also increased in the KA group, and this potentiation was reversed by both $2 \mathrm{~Hz}$ ES and $15 \mathrm{~Hz}$ ES, except in sham controls (Figure 6). All data were analyzed and plotted as Figure 7.

\section{Discussion}

The current study provides information about the molecular effects of ES on TLR4-related mechanisms in KA-induced epileptic rats. Several articles have suggested that brain inflammation is an intrinsic feature of epileptic seizures [25]. 


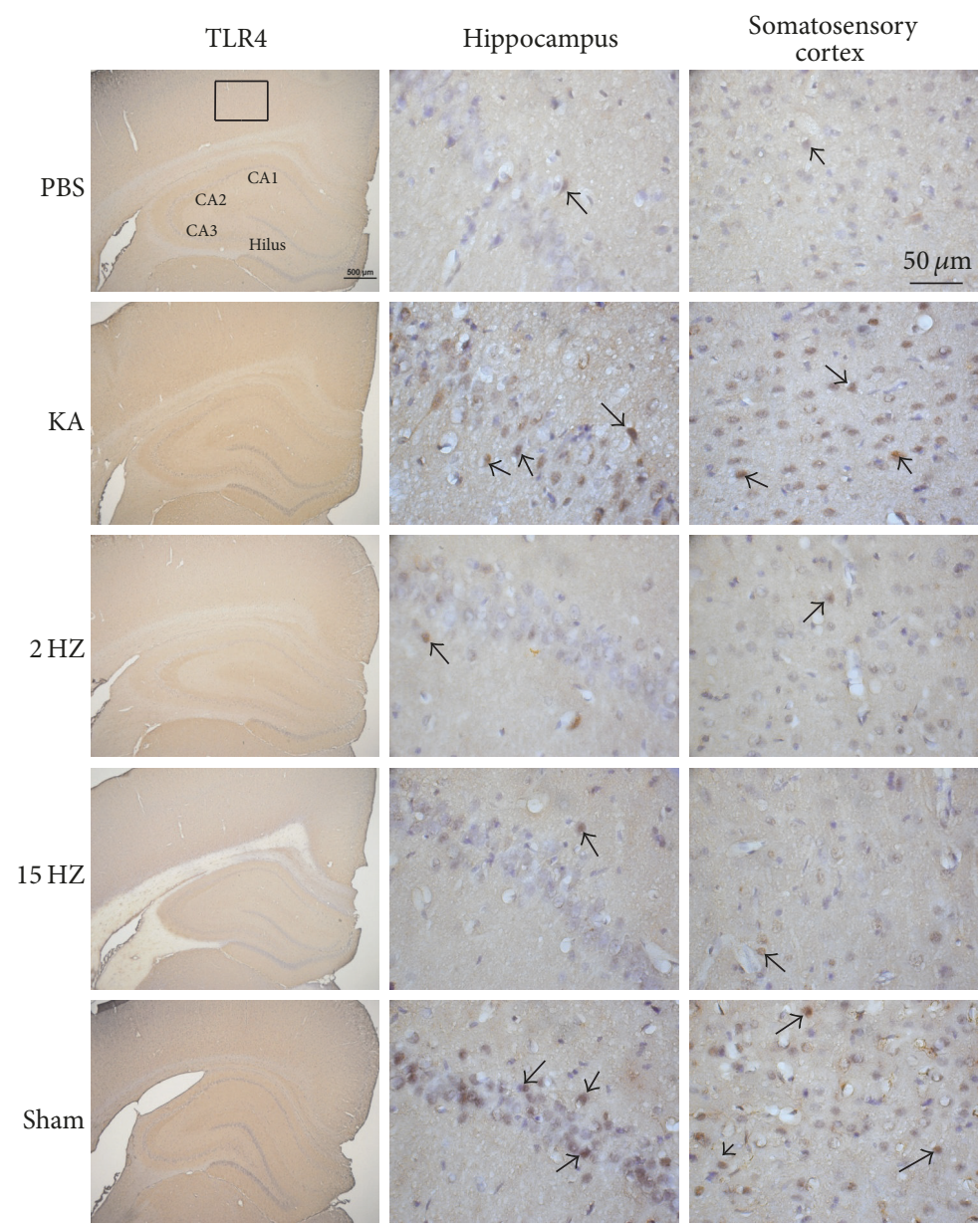

FIGURE 4: Expressions of TLR4 in the hippocampus and somatosensory cortex of PBS, KA, $2 \mathrm{~Hz}$ ES, $15 \mathrm{~Hz}$ ES, and sham rats. Arrows identify immunopositive neurons.

In inflammatory conditions of CNS, TLR can be activated by inflammatory mediators such as interleukin-1 (IL-1) and high-mobility group box-1 (HMGB1). Activation of TLR further triggers a signaling pathway comprising $\mathrm{cAMP}, \mathrm{NF} \kappa \mathrm{B}$, and COX-2 [25]. Song et al. reported that both the mRNA and protein expression of TLR4 in rat hippocampus were reliably increased with pentylenetetrazole injection [26]. They suggested that TLR4 contributes to epilepsy and may also contribute to epileptic therapy [26]. Luan et al. reported that HMGB1 was detected in the cytoplasm of astrocytes in the cortex, suggesting that it is released by glial cells [27]. Levels of HMGB1 and its receptors were increased in inflammatory conditions of the cortex. In brain inflammation, stronger TLR was observed in gray matter [28]. A recent article reported that activation of TLR4, which is determined by agonist injection, influences neuronal excitability to recruit sterile inflammatory responses [29]. We previously suggested that COX-2 levels in the hippocampus and the number of COX-2 immunoreactive cells in the hippocampal CA1 region increased after KA-induced epileptic seizures, and this increase was reduced through a 6-week application of ES at the ear or EA at the ST36-ST37 acupoints [3]. This has an anti-inflammatory effect, which can reduce COX-2 overexpression, suggesting that ES and EA are beneficial for the treatment of epileptic seizures [3]. Our results indicated that $2 \mathrm{~Hz}$ or $15 \mathrm{~Hz}$ ES successfully controlled epileptic seizures by regulating the TLR4 signaling pathway.

Han reported that there are four endogenous opioids, namely, $\beta$-endorphin, enkephalin, endomorphin, and dynorphin that can be released by EA in the brain [22]. They indicated that $\beta$-endorphin is released mainly around the periaqueductal gray matter at $2 \mathrm{~Hz}$ and $15 \mathrm{~Hz}$ EA. In addition, enkephalin and endomorphin increased majorly in the spinal cord by $2 \mathrm{~Hz}$ EA. Furthermore, dynorphin is released at the spinal cord level but only under high frequency EA. EA is suggested to be involved in the inflammatory response through the hypothalamus-pituitary-adrenal axis and the nervous system [30]. EA is also indicated to reduce the inflammation-induced expression of neurokinin-1 in the spinal cord dorsal horn (SCDH) of rats [31]. Activation of opioid receptors markedly reduces peripheral inflammation in local tissues [32] and suppresses $\mathrm{Ca}^{2+}$ currents in primary afferent neurons [33]. Several reports suggest that TLR4 plays a crucial role in epilepsy $[27,29,34]$. Accordingly, we suggest that ES increases the level of opiates in both peripheral and central levels to reduce TLR4 expression through inhibition 

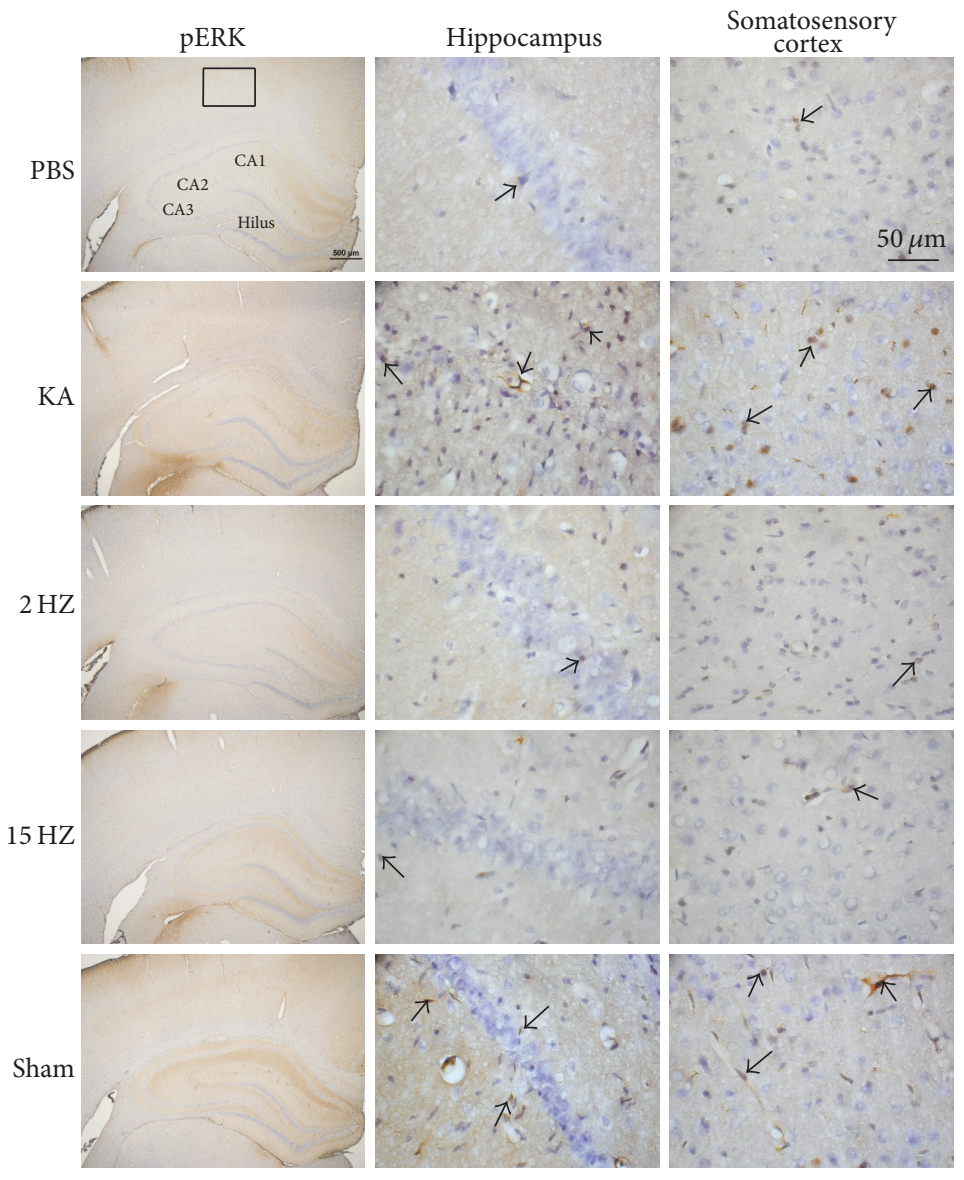

FIGURE 5: Expressions of pERK in the hippocampus and somatosensory cortex of PBS, KA, $2 \mathrm{~Hz}$ ES, $15 \mathrm{~Hz}$ ES, and sham rats. Arrows identify immunopositive neurons.

of inflammatory responses. In Chinese medicine, ES or EA is usually used to treat epilepsy. Auricular sensory afferents constituted by vagus, glossopharyngeal, and facial nerves can contribute to parasympathetic nerve activity [35-37]. Vagus nerve stimulation has been used to reduce the occurrence of seizures in pediatric patients with intractable epilepsy [37, 38]. ES has also been reported to activate parasympathetic tone to reduce epilepsy [38]. Recently, acupuncture is reported to reduce hippocampal neuronal death through inhibition of inflammatory mediators in epileptic KA-induced mice [3]. Our recent study reported that $\mathrm{pERK1/2}$ expression was increased in the KA-induced seizure rats and can be further reduced by both auricular and somatic EA at 6 weeks after induction [39]. Li et al. also indicated that spontaneous seizures and associated ERK activation could contribute to the proliferation in epilepsy model [40]. In addition, recent article showed that $\mathrm{NF} \kappa \mathrm{B}$ signaling pathway is crucial in a spikewave discharges (SWD) characterizing absence epilepsy in WAG/Rij rats [41]. We suggested that $2 \mathrm{~Hz}$ or $15 \mathrm{~Hz}$ ES successfully controlled epileptic seizures by attenuating the pERK and $\mathrm{pNF} \kappa \mathrm{B}$ signaling pathway.

In conclusion, ES treatment leads to a decrease in inflammatory TLR4 expression in the prefrontal cortex, hippocampus, and cerebral cortex of epileptic rats. Furthermore,
pCaMKII $\alpha$, pERK, pp38, and pJNK are involved in this inflammatory process. Moreover, transcriptional $\mathrm{pNK} \kappa \mathrm{B}$ levels in the three brain areas also were altered by 2 or $15 \mathrm{~Hz}$ ES. Our data strongly demonstrate an anti-inflammatory effect of ES in KA-induced epileptic rats. Thus, the knowledge obtained here supports its use in the treatment of epilepsy.

\section{Conflicts of Interest}

The authors declare that there are no conflicts of interest associated with this manuscript and there is no significant financial support to influence the outcome.

\section{Authors' Contributions}

E.-T. Liao and C.-P. Huang performed the animal experiments and wrote the manuscript text; Y.-W. Lin and N.Y. Tang participated in discussion and provided help; C.L. Hsieh participated in protocol design and revised the manuscript text. All authors have reviewed and approved the manuscript. 


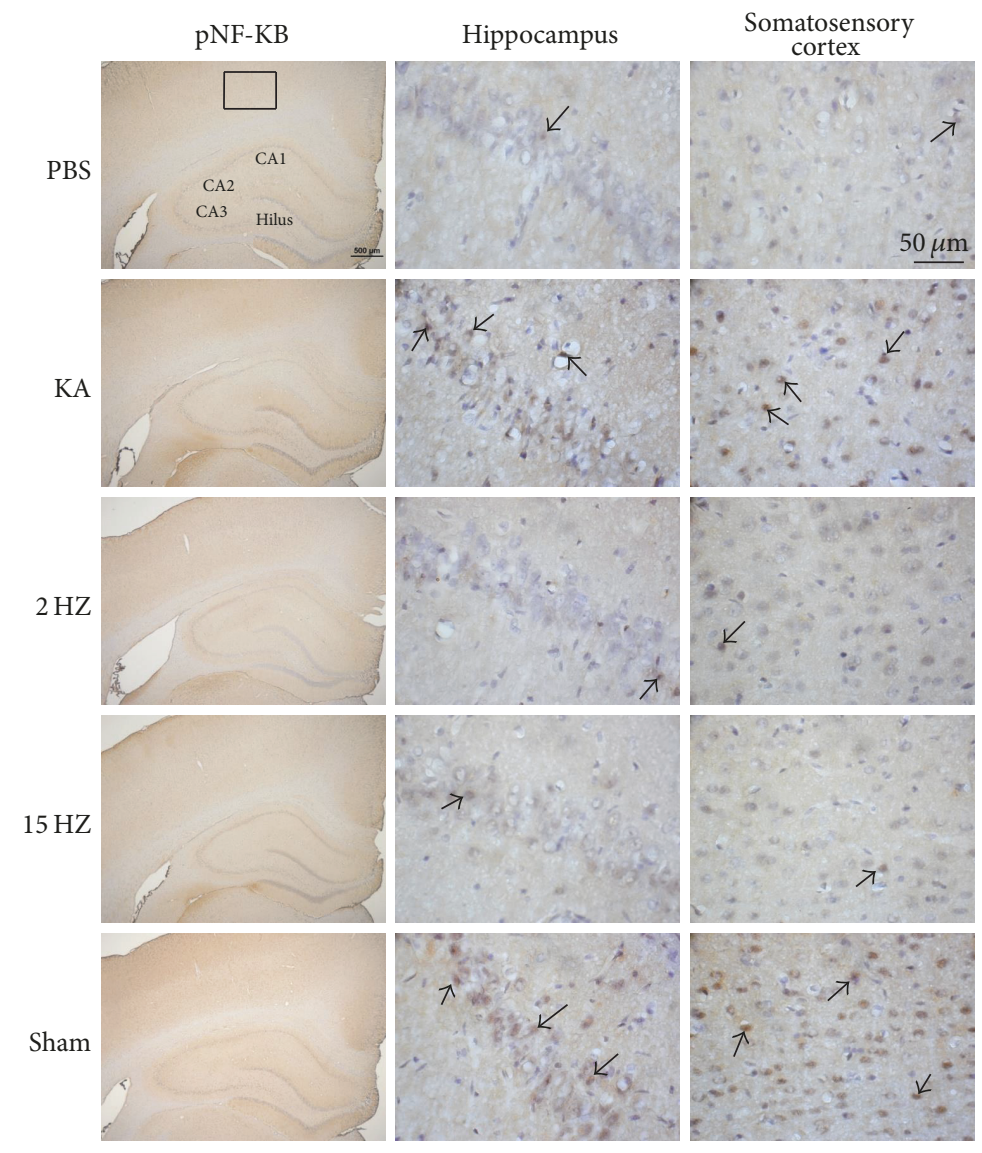

FIGURE 6: Expressions of pNFkB in the hippocampus and somatosensory cortex of PBS, KA, $2 \mathrm{~Hz}$ ES, $15 \mathrm{~Hz}$ ES, and sham rats. Arrows identify immunopositive neurons.

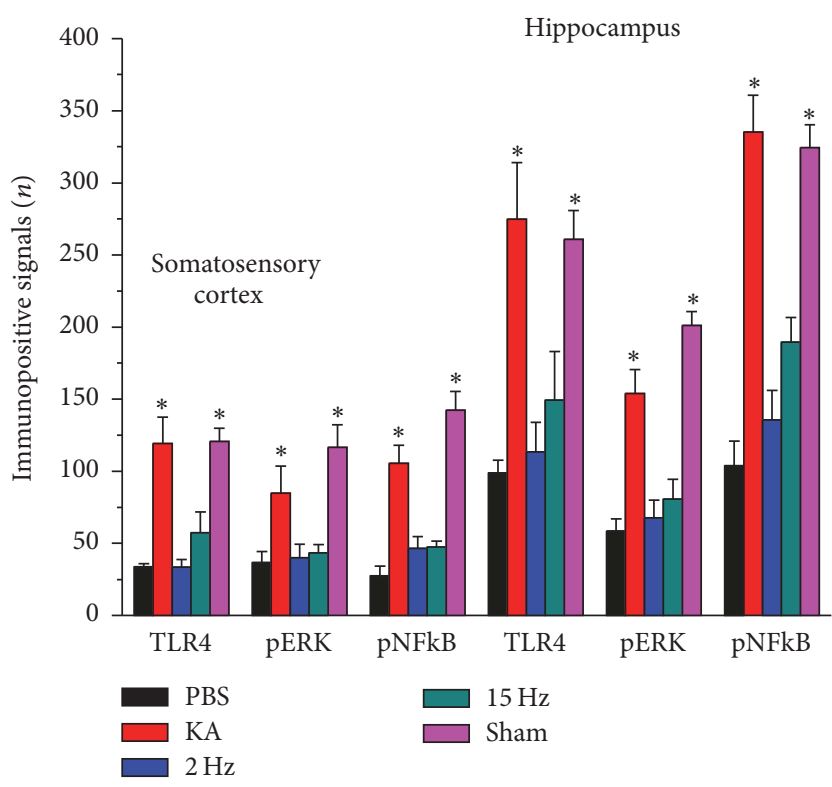

FIGURE 7: Immunopositive signals of TLR4, pERK, and $\mathrm{pNF} \kappa \mathrm{B}$ in the hippocampus and somatosensory cortex of PBS, $\mathrm{KA}, 2 \mathrm{~Hz}$ ES, $15 \mathrm{~Hz}$ ES, and sham rats. The immunopositive signals of TLR4, pERK, and $\mathrm{pNF} \kappa \mathrm{B}$ from hippocampus and somatosensory cortex were presented in each group. ${ }^{*} p<0.05$, as compared to PBS group.

\section{Acknowledgments}

This study was supported by the Minister of Science and Technology, Taiwan (Grant no. MOST-105-2320-B-039039-016), in part by the Taiwan Ministry of Health and Welfare Clinical Trial and Research Center of Excellence (MOHW106-TDU-B-212-133004), and by CMU under the Aim for Top University Plan of the Ministry of Education, Taiwan.

\section{References}

[1] R. Moavero, M. E. Santarone, C. Galasso, and P. Curatolo, "Cognitive and behavioral effects of new antiepileptic drugs in pediatric epilepsy," Brain \& Development, vol. 39, no. 6, pp. 464-469, 2017.

[2] L. Lagae, "The importance of assessing behaviour and cognition in antiepileptic drug trials in children and adolescents," Acta Neurologica Belgica, vol. 117, no. 2, pp. 425-432, 2017.

[3] E.-T. Liao, N.-Y. Tang, Y.-W. Lin, and C. Liang Hsieh, "Longterm electrical stimulation at ear and electro-acupuncture at ST36-ST37 attenuated COX-2 in the CA1 of hippocampus in kainic acid-induced epileptic seizure rats," Scientific Reports, vol. 7, no. 1, article no. 472, 2017. 
[4] N. D. Schartz, S. A. Herr, L. Madsen et al., "Spatiotemporal profile of Map2 and microglial changes in the hippocampal CA1 region following pilocarpine-induced status epilepticus," Scientific Reports, vol. 6, no. 1, 2016.

[5] H. Yoshida, M. Nishikawa, S. Yasuda et al., "Fibronectin inhibits cytokine production induced by CpG DNA in macrophages without direct binding to DNA," Cytokine, vol. 60, no. 1, pp. 162170, 2012.

[6] A. Gross, F. Benninger, R. Madar et al., “Toll-like receptor 3 deficiency decreases epileptogenesis in a pilocarpine model of SEinduced epilepsy in mice," Epilepsia, vol. 58, no. 4, pp. 586-596, 2017.

[7] V. Iori, A. M. Iyer, T. Ravizza et al., "Blockade of the IL-1R1/TLR4 pathway mediates disease-modification therapeutic effects in a model of acquired epilepsy," Neurobiology of Disease, vol. 99, pp. 12-23, 2017.

[8] M. Maroso, S. Balosso, T. Ravizza et al., "Toll-like receptor 4 and high-mobility group box-1 are involved in ictogenesis and can be targeted to reduce seizures," Nature Medicine, vol. 16, no. 4, pp. 413-419, 2010.

[9] E. Zurolo, A. Iyer, M. Maroso et al., "Activation of toll-like receptor, RAGE and HMGB1 signalling in malformations of cortical development," Brain, vol. 134, no. 4, pp. 1015-1032, 2011.

[10] C. Li, Y. Yu, W. Li et al., "Phycocyanin attenuates pulmonary fibrosis via the TLR2-MyD88-NF- $\kappa$ B signaling pathway," Scientific Reports, vol. 7, no. 1, article no. 5843, 2017.

[11] X. Feng, Y. Peng, M. Liu, and L. Cui, "Dl-3-n-butylphthalide extends survival by attenuating glial activation in a mouse model of amyotrophic lateral sclerosis," Neuropharmacology, vol. 62, no. 2, pp. 1004-1010, 2012.

[12] H. Gao, X. Liu, W. Sun et al., "Total tanshinones exhibits antiinflammatory effects through blocking TLR4 dimerization via the MyD88 pathway," Cell Death \& Disease, vol. 8, no. 8, p. e3004, 2017.

[13] T. B. Nielsen, P. Pantapalangkoor, J. Yan et al., "Diabetes exacerbates infection via hyperinflammation by signaling through TLR4 and RAGE," mBio, vol. 8, no. 4, Article ID e00818-17, 2017.

[14] X. Chen, S. Wu, C. Chen et al., "Omega-3 polyunsaturated fatty acid supplementation attenuates microglial-induced inflammation by inhibiting the HMGB1/TLR4/NF-KB pathway following experimental traumatic brain injury," Journal of Neuroinflammation, vol. 14, no. 1, article no. 143, 2017.

[15] A. Das, S. Arifuzzaman, T. Yoon et al., "RNA sequencing reveals resistance of TLR4 ligand-activated microglial cells to inflammation mediated by the selective jumonji H3K27 demethylase inhibitor," Scientific Reports, vol. 7, no. 1, article no. 6554, 2017.

[16] C.-T. Kuo, Y.-W. Lin, N.-Y. Tang, C.-Y. Cheng, and C.-L. Hsieh, "Electric stimulation of the ears ameliorated learning and memory impairment in rats with cerebral ischemia-reperfusion injury," Scientific Reports, vol. 6, Article ID 20381, 2016.

[17] M. Choowanthanapakorn, K.-W. Lu, J. Yang, C.-L. Hsieh, and Y.-W. Lin, "Targeting TRPV1 for Body Weight Control using TRPV1-/-Mice and Electroacupuncture," Scientific Reports, vol. 5, Article ID 17366, 2015.

[18] K.-W. Lu, J. Yang, C.-L. Hsieh, Y.-C. Hsu, and Y.-W. Lin, "Electroacupuncture restores spatial learning and downregulates phosphorylated N-methyl-Daspartate receptors in a mouse model of Parkinson's disease," Acupuncture in Medicine, vol. 35, no. 2, pp. 133-141, 2017.

[19] R.-Z. Kou, H. Chen, M.-L. Yu, T.-C. Xu, S.-P. Fu, and S.F. Lu, "Acupuncture for behavioral changes of experimental depressive disorder: A systematic review and meta-analysis," Scientific Reports, vol. 7, no. 1, article no. 9669, 2017.

[20] H.-Y. Liao, C.-L. Hsieh, C.-P. Huang, and Y.-W. Lin, "Electroacupuncture Attenuates CFA-induced Inflammatory Pain by suppressing Nav1.8 through S100B, TRPV1, Opioid, and Adenosine Pathways in Mice," Scientific Reports, vol. 7, Article ID 42531, 2017.

[21] K.-W. Lu, C.-L. Hsieh, J. Yang, and Y.-W. Lin, "Effects of electroacupuncture in a mouse model of fibromyalgia: role of $\mathrm{N}$ methyl-D-aspartate receptors and related mechanisms," Acupuncture in Medicine, vol. 35, no. 1, pp. 59-68, 2017.

[22] J. S. Han, "Acupuncture: neuropeptide release produced by electrical stimulation of different frequencies," Trends in Neurosciences, vol. 26, no. 1, pp. 17-22, 2003.

[23] R. Zhang, L. Lao, K. Ren, and B. M. Berman, "Mechanisms of acupuncture-electroacupuncture on persistent pain," Anesthesiology, vol. 120, no. 2, pp. 482-503, 2014.

[24] N. Goldman, M. Chen, T. Fujita et al., "Adenosine A1 receptors mediate local anti-nociceptive effects of acupuncture," Nature Neuroscience, vol. 13, no. 7, pp. 883-888, 2010.

[25] A. Vezzani, E. Aronica, A. Mazarati, and Q. J. Pittman, "Epilepsy and brain inflammation," Experimental Neurology, vol. 244, pp. 11-21, 2013.

[26] M.-Y. Song, F.-F. Tian, H. Liu et al., "Expression of SOCSs and TLRs in the hippocampus of pentylenetetrazole kindling model," Clinical Laboratory, vol. 60, no. 2, pp. 233-240, 2014.

[27] G. Luan, Q. Gao, F. Zhai, Y. Chen, and T. Li, "Upregulation of HMGB1, toll-like receptor and RAGE in human Rasmussen's encephalitis," Epilepsy Research, vol. 123, pp. 36-49, 2016.

[28] J.-S. Medel-Matus, A. Reynolds, D. Shin, R. Sankar, and A. Mazarati, "Regulation of kindling epileptogenesis by hippocampal Toll-like receptors 2," Epilepsia, vol. 58, no. 8, pp. e122-e126, 2017.

[29] Y. Li, A. A. Korgaonkar, B. Swietek et al., "Toll-like receptor 4 enhancement of non-NMDA synaptic currents increases dentate excitability after brain injury," Neurobiology of Disease, vol. 74, no. 22, pp. 240-253, 2015.

[30] J. Q. Fang, J. F. Fang, Y. Liang, and J. Y. Du, “Electroacupuncture mediates extracellular signal-regulated kinase $1 / 2$ pathways in the spinal cord of rats with inflammatory pain," BMC Complementary \& Alternative Medicine, vol. 14, no. 1, article 285, 2014.

[31] Y. Wang, D. Hackel, F. Peng, and H. L. Rittner, "Long-term antinociception by electroacupuncture is mediated via peripheral opioid receptors in free-moving rats with inflammatory hyperalgesia," European Journal of Pain, vol. 17, no. 10, pp. 14471457, 2013.

[32] I. Vetter, D. Kapitzke, S. Hermanussen, G. R. Monteith, and P. J. Cabot, "The Effects of $\mathrm{pH}$ on Beta-Endorphin and Morphine Inhibition of Calcium Transients in Dorsal Root Ganglion Neurons," The Journal of Pain, vol. 7, no. 7, pp. 488-499, 2006.

[33] N. P. Turrin and S. Rivest, "Innate immune reaction in response to seizures: implications for the neuropathology associated with epilepsy," Neurobiology of Disease, vol. 16, no. 2, pp. 321-334, 2004.

[34] G. Lachuriya, R. K. Garg, A. Jain et al., "Toll-like Receptor4 Polymorphisms and Serum Matrix Metalloproteinase-9 in Newly Diagnosed Patients with Calcified Neurocysticercosis and Seizures," Medicine (United States), vol. 95, no. 17, Article ID e3288, 2016. 
[35] W. He, X.-H. Jing, B. Zhu et al., "The auriculo-vagal afferent pathway and its role in seizure suppression in rats," BMC Neuroscience, vol. 14, article 85, 2013.

[36] W. He, P.-J. Rong, L. Li, H. Ben, B. Zhu, and G. Litscher, "Auricular acupuncture may suppress epileptic seizures via activating the parasympathetic nervous system: A hypothesis based on innovative methods," Evidence-Based Complementary and Alternative Medicine, vol. 2012, Article ID 615476, 2012.

[37] A. C. Vivas, C. J. Reitano, H. Waseem, S. R. Benbadis, and F. L. Vale, "An analysis of quality of life (QOL) in patients with epilepsy and comorbid psychogenic nonepileptic seizures (PNES) after vagus nerve stimulation (VNS)," Epilepsy \& Behavior, vol. 73, pp. 208-213, 2017.

[38] H. Liu, Z. Yang, L. Huang, W. Qu, H. Hao, and L. Li, "Heart-rate variability indices as predictors of the response to vagus nerve stimulation in patients with drug-resistant epilepsy," Epilepsia, vol. 58, no. 6, pp. 1015-1022, 2017.

[39] Y. W. Lin and C. L. Hsieh, "Auricular electroacupuncture reduced inflammation-related epilepsy accompanied by altered TRPA1, $\mathrm{pPKC} \alpha, \mathrm{pPKC} \varepsilon$, and $\mathrm{pERk1/2}$ signaling pathways in kainic acid-treated rats," Mediators of Inflammation, vol. 2014, Article ID 493480, 9 pages, 2014.

[40] Y. Li, Z. Peng, B. Xiao, and C. R. Houser, "Activation of ERK by spontaneous seizures in neural progenitors of the dentate gyrus in a mouse model of epilepsy," Experimental Neurology, vol. 224, no. 1, pp. 133-145, 2010.

[41] B. Gyorffy, Z. Kovacs, P. Gulyassy et al., "Brain protein expression changes in WAG/Rij rats, a genetic rat model of absence epilepsy after peripheral lipopolysaccharide treatment," Brain, Behavior, and Immunity, vol. 35, pp. 86-95, 2014. 


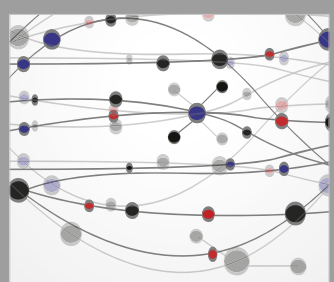

The Scientific World Journal
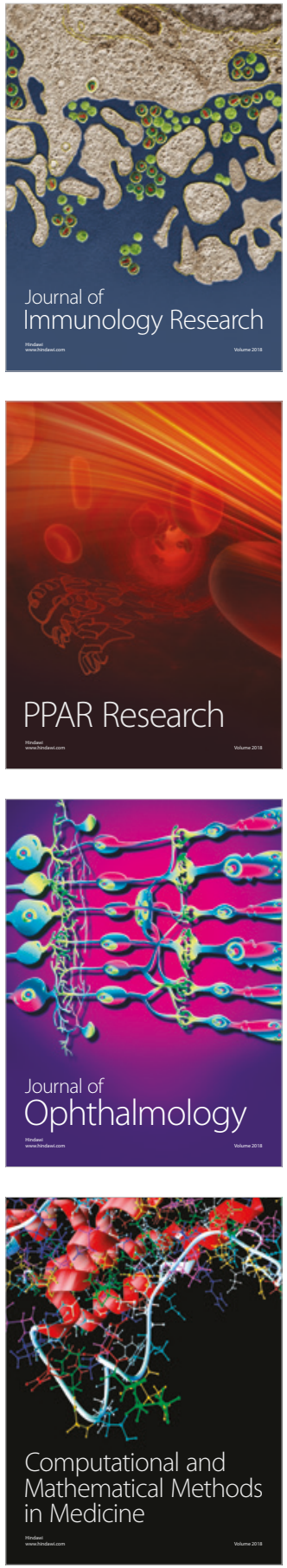

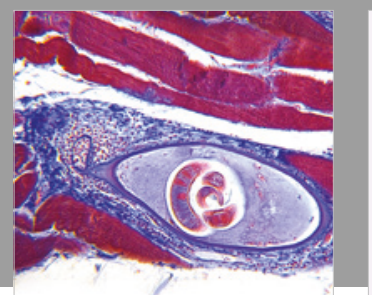

Gastroenterology Research and Practice

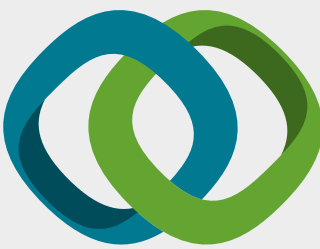

\section{Hindawi}

Submit your manuscripts at

www.hindawi.com
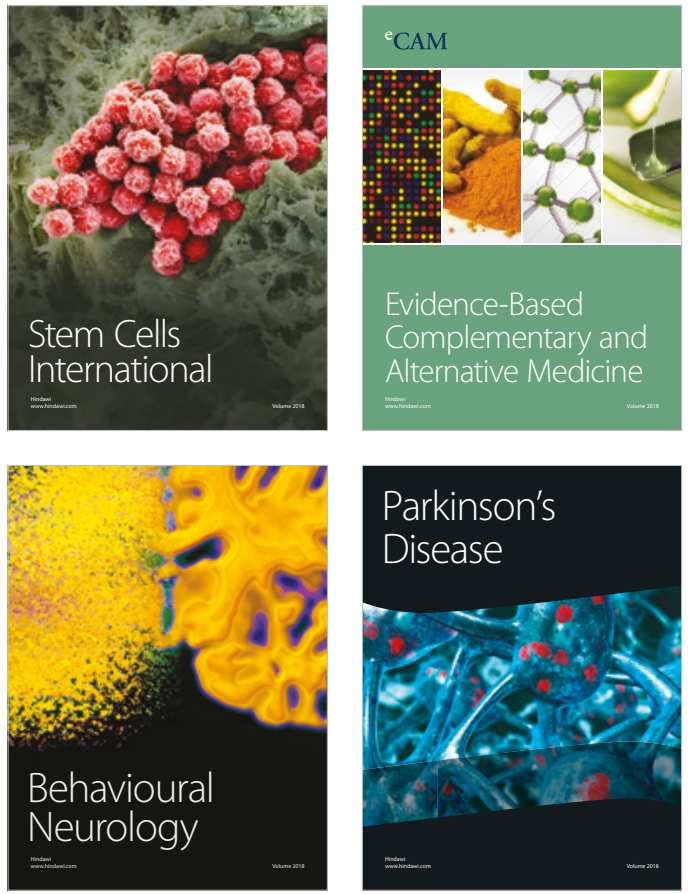

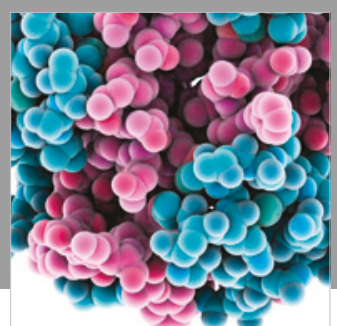

ournal of

Diabetes Research

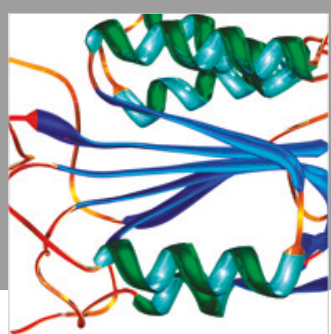

Disease Markers
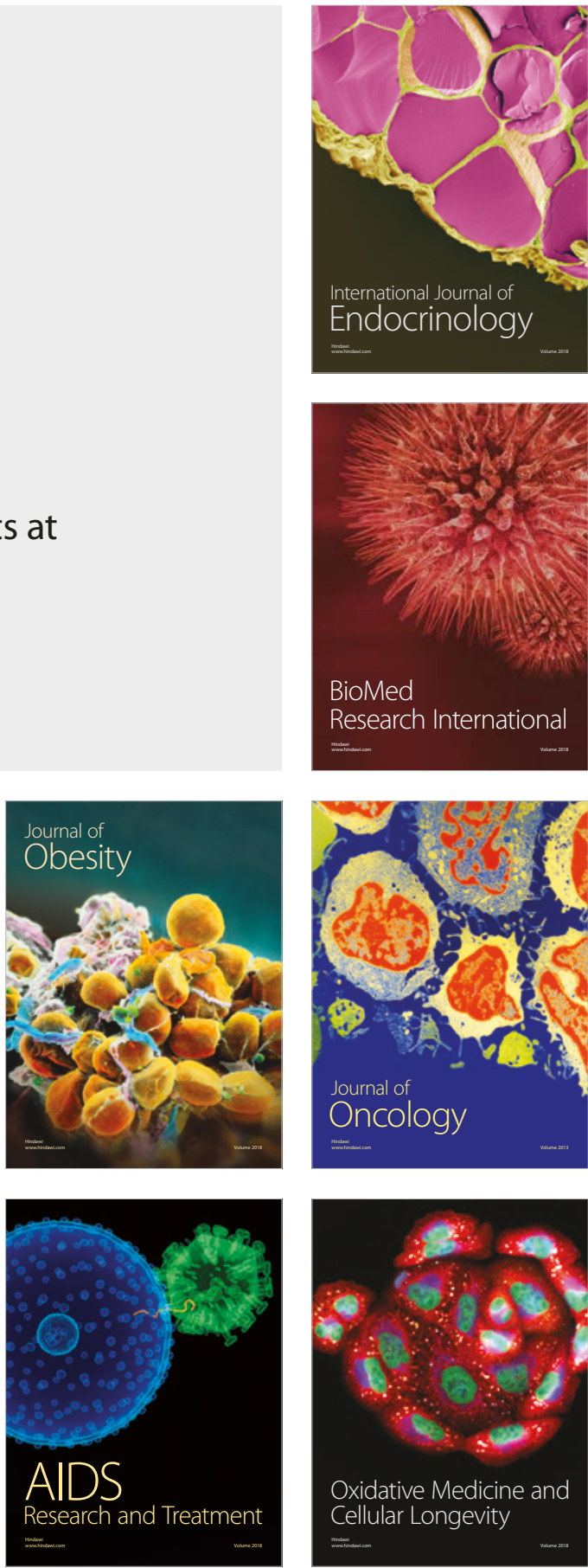